\title{
AN EXTERIOR INTERFACE PROBLEM IN TWO-DIMENSIONAL ELASTODYNAMICS*
}

\author{
By \\ JACOBO BIELAK and R. C. MACCAMY \\ Carnegie-Mellon University
}

1. Introduction. In this paper we present and analyze a method of solution for an interface problem in linear elasticity. We deal with the special situation of steady-state, time-harmonic, two-dimensional anti-plane strain. The physical problem is this. Suppose one has a half-space of homogeneous, isotropic, linearly elastic material (the earth) bounded by a traction-free plane. In this material we have an elastic wave, incident plus reflected, which is time-periodic with frequency $\omega$. Suppose now that a cylindrical obstacle of different and possibly inhomogeneous material is inserted in the free surface. The problem is to determine the time-periodic steady-state limit for the total fields inside and outside the obstacle.

We refer to [1] for a general discussion of problems of the above type. For linearly elastic, isotropic materials the fields are displacement $\mathbf{U}$, strain $\left(\mathbb{E}=\frac{1}{2}\left(\nabla \mathbf{U}+(\nabla \mathbf{U})^{t}\right)\right.$ and the stress $\mathfrak{J}$,

$$
\mathfrak{J}=\lambda(\operatorname{div} \mathbf{U}) \mathbf{I}+2 \mu \mathfrak{E} .
$$

In general $\lambda$ and $\mu$ are functions of position; the material is homogeneous if $\lambda$ and $\mu$ are constant. The equation of motion is

$$
\rho \mathbf{U}_{t t}=\operatorname{div} \mathfrak{J}+\mathbf{b}
$$

where $\rho$ is density and $\mathbf{b}$ is body force.

Consider the geometry of Fig. 1. The region $\Omega_{+}^{\prime}$ is to represent homogeneous elastic material with $\lambda_{+}, \mu_{+}, \rho_{+}$. The region $\Omega^{\prime}$ represents a (possibly inhomogeneous) cylinder with $\lambda_{-}, \mu_{-}, \rho_{-}$depending on $x_{1}$ and $x_{2} \cdot x_{2}=0$ is a traction-free surface. $\Gamma^{\prime}$ is the boundary of $\Omega^{\prime}$.

The anti-plane strain (SH wave) situation for Fig. 1 occurs when the displacement has the form

$$
\mathrm{U}=\mathrm{U}\left(x_{1}, x_{2}, t\right) \mathbf{e}_{3} .
$$

For such fields $\mathfrak{E}_{13}=\mathfrak{E}_{31}=\frac{1}{2} U_{x_{1}}, \mathfrak{E}_{23}=\mathfrak{E}_{32}=\frac{1}{2} U_{x_{2}}, \mathfrak{F}_{13}=\mathfrak{F}_{31}=\mu U_{x_{1}}, \mathfrak{F}_{23}=\mathfrak{F}_{32}=$ $\mu U_{x_{2}}$ and all other strain and stress components are zero. In the absence of body forces Eq. (1.2) reduces to

$$
L_{\mu} U=\left(\mu U_{x_{1}}\right)_{x_{1}}+\left(\mu U_{x_{2}}\right)_{x_{2}}=\rho U_{t t} .
$$

The fact that $x_{2}=0$ is traction-free yields

$$
U_{x_{2}}\left(x_{1}, 0\right)=0 .
$$

\footnotetext{
* Received May 24, 1982. This work was supported in part by the National Science Foundation under Grant MCS-8001944.
} 
Across the interface $\Gamma^{\prime}$ the displacement and the traction are continuous, that is,

$$
U^{+}=U^{-}, \quad \mu_{-} U_{n}^{-}=\mu_{+} U_{n}^{+} \text {on } \Gamma^{\prime} \text {. }
$$

The plus and minus denote limits from $\Omega_{+}^{\prime}$ and $\Omega^{\prime}$ respectively and $\mathbf{n}$ is the normal.

We assume that the displacements are time-periodic of the form

$$
U(x, t)=\operatorname{Re}\left(u(x) e^{i \omega t}\right),
$$

so that (1.4) becomes

$$
L_{\mu} u+\omega^{2} \rho u=0 .
$$

We let $u^{0}(x)$ correspond to the driving field. $u^{0}$ will consist of an incoming wave $v^{0}$, defined in all of $\mathbb{R}^{2}$, and its reflection, that is

$$
u^{0}\left(x_{1}, x_{2}\right)=v^{0}\left(x_{1}, x_{2}\right)+v^{0}\left(x_{1},-x_{2}\right) .
$$

We nondimensionalize the problem. We choose a length scale $L^{2}=\mu_{+} / \rho_{+} \omega^{2}$, replace $x$ by $x / L$ and introduce the parameters

$$
\mu=\mu_{-} / \rho_{+} \omega^{2} L^{2}, \quad \beta^{2}=\rho_{-} / \rho_{+} .
$$

Then (1.8) and (1.6) yield

$$
\begin{aligned}
L_{\mu} u+\beta^{2} u & =0 \quad \text { in } \quad \Omega^{\prime}, \quad \Delta u+u=0 \quad \text { in } \Omega_{+}^{\prime}, \\
u^{-} & =u^{+}, \quad \mu u_{n}^{-}=u_{n}^{+} \quad \text { on } \Gamma^{\prime} .
\end{aligned}
$$

We set $w=u$ in $\Omega^{\prime}$ and $w=u-u^{0}$ in $\Omega_{+}^{\prime}$ so that $w$ represents the scattered wave in $\Omega_{+}^{\prime}$. We require that $w$ satisfy the radiation condition

$$
w \sim r^{-1 / 2} e^{-i r} \text { as } r=|x| \rightarrow \infty_{-}
$$

We have then the following.

Problem $\left(\mathrm{P}^{\prime}\right)$. Find $w$ satisfying (1.13) and such that

$$
\begin{array}{rlrl}
L_{\mu} w+\beta^{2} w & =0 \quad \text { in } \quad \Omega^{\prime}, & \Delta w+w=0 \text { in } \Omega_{+}^{\prime}, \\
w^{-} & =w^{+}+u^{0}, \quad \mu w_{n}^{-}=w_{n}^{+}+u_{n}^{0} .
\end{array}
$$

There exists a number of procedures for solving problems like (1.14). In the elasticity

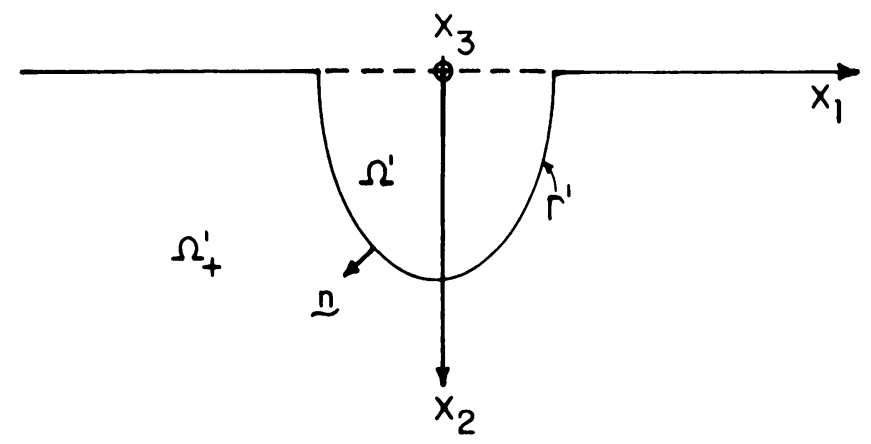

FIG. 1 
setting we refer to [1, 2, 3]. A more general class of interface problems is discussed in [4] with a model problem analyzed theoretically in [5]. Our analysis has three stages, as outlined in Sec. 2. Our first step is to introduce two different equivalent problems $\left(\mathbf{P}_{\mathbf{0}}\right)$ and $\left(P_{1}\right)$ in each of which we work only in $\Omega^{\prime}$ but have to introduce an auxiliary function $\varphi$ on $\Gamma^{\prime}$. Next we rephrase $\left(\mathbf{P}_{0}\right)$ and $\left(\mathrm{P}_{1}\right)$ as variational problems $\left(\mathrm{VP}_{0}\right)$ and $\left(\mathrm{VP}_{1}\right)$ in which all boundary conditions are natural. Then we introduce finite-dimensional approximations $\left(\mathrm{AVP}_{0}\right)$ and $\left(\mathrm{AVP}_{1}\right)$.

One has a choice of which of the problems $\left(\mathbf{P}_{0}\right)$ or $\left(\mathbf{P}_{1}\right)$ to solve. As indicated in Sec. 2, $\left(\mathrm{P}_{0}\right)$ is somewhat preferable if one is primarily interested in $\Omega^{\prime}$ and $\left(P_{1}\right)$ if the emphasis is on $\Omega_{+}^{\prime}$. The main reason for introducing the two is that they are "adjoint" in the sense that to analyze the procedure for either one must have information about the other.

Remark. We indicate in Sec. 2 that there is actually a whole family of auxiliary problems $\mathrm{P}_{\alpha}, 0 \leq \alpha \leq 1$ and that when $\alpha=\frac{1}{2}$ the resulting problem is "symmetric" in a sense made precise there. For $0<\alpha<1$, however, we need two boundary functions.

Our methods are all in the spirit of [4] and [5], a combination of finite-element and boundary integral methods. In particular, our $\left(\mathbf{P}_{0}\right)$ is an extension to a more complicated situation of the problem studied in [5].

In Sec. 3 we give an analysis of our auxiliary problems and in Sec. 4 we discuss convergence of the finite-dimensional approximations. There is a complication here with which we do not deal in this paper. The complication is the confluence of $\Gamma^{\prime}$ with $x_{2}=0$. This produces singularities in the fields and these affect the analysis. We will suppress this difficulty here by a symmetrizing process as we describe now.

We make three simplifying assumptions. The first is that the curve $\Gamma^{\prime}$ is perpendicular to $x_{2}=0$. Then we can reflect $\Gamma^{\prime}, \Omega^{\prime}$ and $\Omega_{+}^{\prime}$ in $x_{2}=0$ to obtain the configuration in all of $\mathbb{R}^{2}$ indicated in Fig. 2 . $\Gamma$ is then a smooth closed curve bounding $\Omega$. Our second assumption is that $\mu$ and $\rho$ satisfy

$$
\mu_{x_{2}}\left(x_{1}, 0\right) \equiv 0, \quad \rho_{x_{2}}\left(x_{1}, 0\right) \equiv 0 .
$$

Then we can extend $\mu$ and $\rho$ to all of $\Omega$ as continuously differentiable functions which are even in $x_{2}$. Observe that $u^{0}$ is defined in all of $\mathbb{R}^{2}$ and, by (1.9), is an even function of $x_{2}$ The problem we actually analyze in Secs. 3 and 4

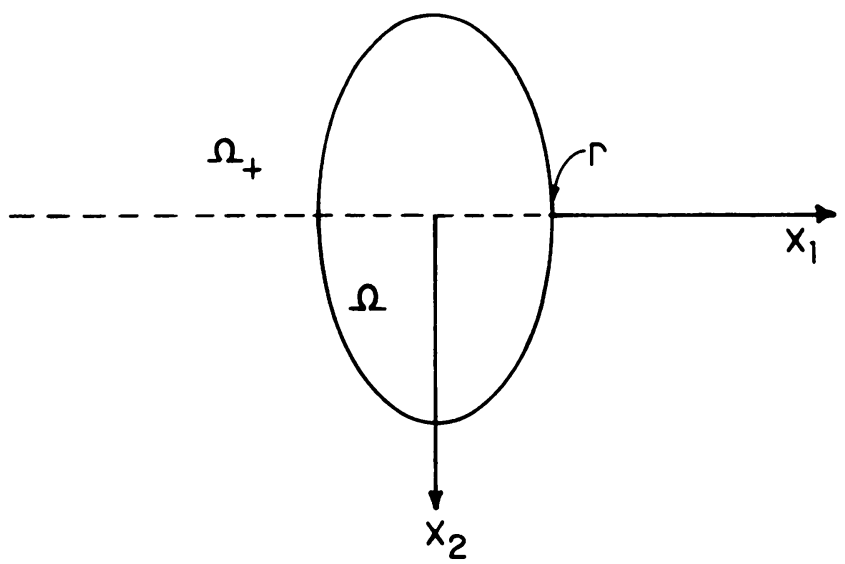

Fig. 2 
Problem (P). Find $w$, satisfying (1.13) in $\Omega_{+}$, such that

$$
\begin{gathered}
L_{\mu} w+\beta^{2} w=0 \quad \text { in } \quad \Omega, \quad \Delta w+w=0 \quad \text { in } \Omega_{+}, \\
w^{-}=w^{+}+u^{0} \quad \mu w_{n}^{-}=w_{n}^{+}+u_{n}^{0} \quad \text { on } \Gamma .
\end{gathered}
$$

Suppose we know that the solution of $(\mathrm{P})$ is unique (to be proved shortly). Then it is easy to verify that the solution of $(\mathrm{P})$ is even in $x_{2}$ and that in $\Omega^{\prime}, \Omega_{+}^{\prime}, w$ will satisfy $\left(\mathrm{P}^{\prime}\right)$.

We remark that problem $(\mathrm{P})$ also occurs in the theory of scattering of electromagnetic waves by dielectric cylinders [6]. Other numerical procedures have been given for this problem, for instance in [6] and [7]. The method in [7] is related to our problem $\left(\mathrm{P}_{0}\right)$.

We present now the quite simple proof of uniqueness of solutions for $(\mathrm{P})$. We denote by $K(\Omega)\left(K\left(\Omega^{+}\right)\right)$the spaces of functions which are twice continuously differentiable in $\Omega$ $\left(\Omega^{+}\right)$and for which $w$ and $\nabla w$ have limits $w^{-}, \nabla w^{-}\left(w^{+}, \nabla w^{+}\right)$on $\Gamma$. For $K\left(\Omega^{+}\right)$we also require (1.13). Then by a solution of $(\mathrm{P})$ we mean $w \in K(\Omega) \cap K\left(\Omega^{+}\right)$satisfying (1.15).

THEOREM 1.1 There exists at most one solution of $(\mathrm{P})$.

Proof: It suffices to prove $u^{0} \equiv 0$ implies $w \equiv 0$. Suppose $w$ is a solution for $u^{0} \equiv 0$. Then by Green's theorem

$$
-\int_{\Omega}\left(\mu\left|\nabla w^{2}\right|^{2}-\beta^{2}|w|^{2}\right) d x+\int_{\Gamma} \mu w_{n}^{-} \bar{w}^{-} d s=0 .
$$

Let $\Omega_{R}$ denote the region $\Omega^{+} \cap(|x|<\mathrm{R})$. Then Green's theorem yields

$$
-\int_{\Omega_{R}}\left(|\nabla w|^{2}-|w|^{2}\right) d x+\int_{\Gamma} w_{n}^{+} \bar{w}^{+} d s+\int_{|x|=\mathbf{R}} w_{n} \bar{w} d s=0 .
$$

By $(1.15)_{3,4}$ the integrals over $\Gamma$ are the same and we obtain

$$
\operatorname{Im} \int_{|x|=R} w_{n} \bar{w} d s=0 .
$$

Eq. (1.18) and a standard argument for exterior problems for the Helmholtz equation (see [8]) imply that $w \equiv 0$ in $\Omega^{+}$. Then $w^{-} \equiv w^{+} \equiv 0$ and $w_{n}^{-} \equiv w_{n}^{+} \equiv 0$ and unique continuation implies that $w \equiv 0$ in $\Omega$.

Let us list the hypotheses under which we operate for the rest of the paper. To avoid technical smoothness assumptions we suppose $\Gamma$ is a $C^{\infty}$ curve and $\mu$ and $\rho$ are (positive) $C^{\infty}$ functions in $\bar{\Omega}$. We need two hypotheses on $\Gamma$ (and $\left.\omega\right)$. These are:

$$
\begin{aligned}
& \Delta v+v=0 \quad \text { in } \quad \Omega, \quad v^{-} \equiv 0 \Rightarrow v \equiv 0 \quad \text { in } \Omega, \\
& L_{\mu} v+\beta^{2} v=0 \quad \text { in } \Omega, \quad \mu w_{n}^{-}=0 \Rightarrow v \equiv 0 \quad \text { in } \Omega \text {. }
\end{aligned}
$$

Hypotheses like these are familiar in boundary-value problems. We observe that, for a given $\Gamma$, there will be infinite sequences $\omega_{k}^{i}, i=1,2, k=1,2,3, \ldots$, for which H.i will fail. We observe that our uniqueness theorem did not require either hypothesis. We believe that they are not necessary for existence either but are simply results of our proof methods.

2. Formulation of solution procedures. In this section we outline our procedures. We present them for $(\mathrm{P})$ with a statement of the modification for $\left(\mathrm{P}^{\prime}\right)$. We begin with some potential theory for the equation

$$
\Delta v+v=0
$$


We define $g$ by the formula

$$
g(x, y)=\frac{i}{4} H_{0}^{(2)}(|x-y|)
$$

where $H_{0}^{(2)}$ is the Hankel function of second kind and order zero. Thus $g$ is the Green's function for (2.1) with (1.13). We define the simple and double layers $\mathscr{S}[\varphi]$ and $\mathscr{D}[\varphi]$ with density $\varphi$ by

$$
\begin{gathered}
\mathscr{S}[\varphi](x)=\int_{\Gamma} \varphi(y) g(x, y) d s_{y}, \\
\mathscr{D}[\varphi](x)=\int_{\Gamma} \varphi(y) \frac{\partial}{\partial n_{y}} g(x, y) d s_{y} .
\end{gathered}
$$

To modify our procedure to $\left(\mathrm{P}^{\prime}\right)$ it is necessary only to replace $g$ by the function.

$$
g(x, y)=\frac{i}{4}\left\{H_{0}^{(2)}(|x-y|)+H_{0}^{(2)}\left(\left|x-y^{*}\right|\right)\right\}
$$

where $y^{*}=\left(y_{1},-y_{2}\right)$. Then $\mathscr{S}$ is the Neumann function in $x_{2}>0$. In all the formulas below one need only replace $\Gamma, \Omega$ and $\Omega_{+}$by $\Gamma^{\prime}, \Omega^{\prime}, \Omega_{+}^{\prime}$.

For smooth functions $\varphi$ the properties of $\mathscr{S}$ and $\mathscr{D}$ are well known. They both satisfy (2.1) in $\Omega$ and in $\Omega^{+}$. We have the following limit relations on $\Gamma$. Define integral operators $S, N, D$ on $\Gamma$ by

$$
\begin{array}{ll}
S[\varphi](x)=\int_{\Gamma} \varphi(y) S(x, y) d s_{y}, & S(x, y)=\left.g(x, y)\right|_{x \in \Gamma}, \\
N[\varphi](x)=\int_{\Gamma} \varphi(y) N(x, y) d s_{y}, & N(x, y)=\left.\frac{\partial g}{\partial n_{x}}(x, y)\right|_{x \in \Gamma}, \\
D[\varphi](x)=\int_{\Gamma} \varphi(y) D(x, y) d s_{y}, & D(x, y)=\left.\frac{\partial g}{\partial n_{y}}(x, y)\right|_{x \in \Gamma} .
\end{array}
$$

Then

$$
\begin{gathered}
\mathscr{S}[\varphi]^{ \pm}=S[\varphi] ; \quad\left(\frac{\partial \mathscr{S}[\varphi]}{\partial n}\right)^{ \pm}= \pm \frac{1}{2} \varphi+N[\varphi] ; \\
(\mathscr{D}[\varphi])^{ \pm}=\mp \frac{1}{2} \varphi+D[\varphi] .
\end{gathered}
$$

The kernel $S$ has a logarithmic singularity while, for smooth curves, the kernels $N$ and D are continuous. Moreover, one has

$$
S(x, y)=S(y, x), \quad N(x, y)=D(y, x) .
$$

We have the following analogues of the Helmholtz formulas. If $v$ satisfies $(2.1)$ in $\Omega$ then

$$
v=\mathscr{D}\left[v^{-}\right]-\mathscr{S}\left[v_{n}^{-}\right] \text {in } \mathbf{\Omega} .
$$

If $v$ satisfies $(2.1)$ and $(1.13)$ in $\Omega^{+}$then

$$
v=\mathscr{S}\left[v_{n}^{+}\right]-\mathscr{D}\left[v^{+}\right] \text {in } \Omega^{+} .
$$

Combining (2.7), (2.8) with (2.5) yields the following results for a $v$ which satisfies $(2.1)$ in $\Omega$ 
or (2.1) and (1.13) in $\Omega^{+}$:

$$
\frac{1}{2} v^{-}=D\left[v^{-}\right]-S\left[v_{n}^{-}\right], \quad \frac{1}{2} v^{+}=S\left[v_{n}^{+}\right]-D\left[v^{+}\right] .
$$

We are now ready to formulate our equivalent problems for $(\mathrm{P})$.

Equivalent problems. Suppose $w$ is a solution of (P). Then we can apply (2.8) and $(2.9)_{2}$. By $(1.15)_{3,4}$ we have $w_{n}^{+}=w_{n}^{-}-u_{n}^{0}$ and $w^{+}=w^{-}-u^{0}$. We obtain then

$$
\begin{gathered}
w=\mathscr{S}\left[\mu w_{n}^{-}-w_{n}^{0}\right]-\mathscr{D}\left[w^{-}-u^{0}\right] \text { in } \Omega^{+}, \\
\frac{1}{2} w^{-}+D\left[w^{-}\right]-S\left[\mu w_{n}^{-}\right]=\frac{1}{2} u^{0}-S\left[u_{n}^{0}\right]+D\left[u^{0}\right] .
\end{gathered}
$$

Now $u^{0}$ is a solution of (2.1) in $\Omega$; hence, by $(2.9)_{1},-S\left[u_{n}^{0}\right]+D\left[u^{0}\right]=\frac{1}{2} u^{0}$, so the right of (2.11) is just $u^{0}$. Let us introduce $\varphi=\mu w_{n}^{-}$as an auxiliary variable. Then (2.11) becomes

$$
\frac{1}{2} w^{-}+D\left[w^{-}\right]-S[\varphi]=u^{0}
$$

and (2.10) becomes

$$
w=\mathscr{S}\left[\varphi-u_{n}^{0}\right]-\mathscr{D}\left[w^{-}-u^{0}\right] \text { in } \Omega^{+} .
$$

We have thus been led to the following problem.

Problem $\mathrm{P}_{0}$. Find $(w, \varphi)$ such that

$$
L_{\mu} w+\beta^{2} w=0 \quad \text { in } \quad \Omega, \quad \mu w_{n}^{-}=\varphi, \quad \frac{1}{2} w^{-}+D\left[w^{-}\right]-S[\varphi]=u^{0} .
$$

Let us obtain a second auxiliary problem. Here we seek $w$ in the form

$$
w=\mathscr{S}[\varphi] \text { in } \Omega^{+} .
$$

From (2.5) we obtain $w^{+}=S[\varphi]$ and $w_{n}^{+}=\frac{1}{2} \varphi+N[\varphi]$. Inserting (1.15) 3,4 yields $w^{-}=$ $S[\varphi]+u^{0}$ and $\mu w_{n}^{-}=\frac{1}{2} \varphi+N[\varphi]+u_{n}^{0}$. This suggests the second problem

Problem $\mathrm{P}_{1}$. Find $(w, \varphi)$ such that

$$
\begin{gathered}
L_{\mu} w+\beta^{2} w=0 \quad \text { in } \Omega, \\
w^{-}=S[\varphi]+u^{0}, \quad \mu w_{n}^{-}=\frac{1}{2} \varphi+N[\varphi]+u_{n}^{0} .
\end{gathered}
$$

Both $\left(P_{0}\right)$ and $\left(P_{1}\right)$ are equivalent to $(P)$. In order to establish this we need a result about the operator $S$. This can be obtained from the work of [9].

Lemma 2.1. Suppose $f \in C^{(1)}(\mathrm{P})$. Then:

(i) There exists a unique $\chi=S^{-1}[f] \in C(\Gamma)$ such that $S[\chi]=f$;

(ii) The (unique) solution of $\Delta v+v=0$ in $\Omega, v=f$ on $\Gamma$ with conditions (1.12) is $v=\mathscr{S}[\chi]$.

THEOREM 2.1 (i). Suppose $w$ is a solution of $(\mathrm{P})$. Then $\left(w, \mu w_{n}^{-}\right)$is a solution of $\left(\mathrm{P}_{0}\right)$.

(ii) Suppose $(w, \varphi)$ is a solution of $\left(\mathrm{P}_{0}\right)$ with $w \in K(\Omega), \varphi \in C(\Gamma)$. Put

$$
w=\mathscr{S}\left[\varphi-u_{n}^{0}\right]-\mathscr{D}\left[w^{-}-u^{0}\right] \text { in } \Omega^{+} .
$$

Then $w$ is a solution of $(\mathrm{P})$.

THEOREM 2.2 (i). Suppose $w$ is a solution of $(\mathrm{P})$. Then $\left(w, S^{-1}\left(w^{-}-u^{0}\right)\right)$ is a solution of $\left(\mathrm{P}_{1}\right)$.

(ii) Suppose $(w, \varphi)$ is a solution of $\left(\mathrm{P}_{1}\right)$ with $w \in K(\Omega), \varphi \in C(\Gamma)$. Put

$$
w=\mathscr{S}[\varphi] \text { in } \Omega^{+} .
$$

Then $w$ is a solution of $(\mathrm{P})$. 
Proof. The proof of (i) in both theorems follows from the calculations made to arrive at $\left(\mathrm{P}_{0}\right)$ and $\left(\mathrm{P}_{1}\right)$ together with the observation that by the lemma $\mathrm{w}^{+}=S[\varphi]$ implies $\varphi=S^{-1}\left[w^{+}\right]$. To establish (ii) for Theorem 2.1 we observe that (2.17) and (2.5) yield

$$
w^{+}=S[\varphi]-D\left[w^{-}\right]+\frac{1}{2}\left(w^{-}-u^{0}\right)-S\left[u_{n}^{0}\right]+D\left[u_{n}^{0}\right] .
$$

But we showed earlier that $-S\left[u_{n}^{0}\right]+D\left[u_{n}^{0}\right]=\frac{1}{2} u^{0}$ and $(2.14)_{3}$ yields $S[\varphi]-D\left[w^{-}\right]=\frac{1}{2} w^{-}-u^{0}$. Hence (2.19) gives $w^{+}=w^{-}-u^{0}$, which is $(1.15)_{3}$. We have then by $(2.17) w=\mathscr{S}\left[\varphi-u_{n}^{0}\right]-\mathscr{D}\left[w^{+}\right]$in $\Omega^{+}$. But by $(2.8) w=\mathscr{S}\left[w_{n}^{+}\right]-\mathscr{D}\left[w^{+}\right]$. Hence $\mathscr{S}\left[\varphi-u_{n}^{0}-w_{n}^{+}\right] \equiv \mathscr{S}\left[\mu w_{n}^{-}-u_{n}^{0}-w_{n}^{+}\right] \equiv 0$ in $\Omega^{+}$. Taking the limit on $\Gamma$ and using Lemma 2.1 (i) yields $\mu w_{n}^{-}=w_{n}^{+}+u_{n}^{0}$, which is $(1.15)_{4}$. The proof of (ii) for Theorem 2.2 is similar.

We will see in the next section that, under appropriate hypotheses, both $\left(\mathrm{P}_{0}\right)$ and $\left(\mathrm{P}_{1}\right)$ have solutions, and we have just seen that both are equivalent to $(\mathrm{P})$. We will give variational formulations of both problems. One has a choice of which to use. We observe that a virtue of $\left(\mathrm{P}_{0}\right)$ is that the quantity $\varphi$ has direct physical meaning; namely, it is $\mu w_{n}^{-}$which determines the traction at the boundary. On the other hand, if one is primarily interested in the exterior field then $\left(\mathrm{P}_{1}\right)$ is preferable since (2.18) is simpler than (2.17). Mathematically both are of interest in the convergence proofs as described in Sec. 4 .

Variational problems. Suppose first that $w$ is a solution of $\left(P_{0}\right)$. We multiply $(2.14)_{1}$ by a test function $\bar{v}$ and integrate over $\Omega$ using the divergence theorem and $(2.14)_{2}$. The result is

$$
-\int_{\Omega}\left(\mu \nabla w \cdot \nabla \bar{v}-\beta^{2} w \bar{v}\right) d x+\int_{\Gamma} \varphi \bar{v} d s=0 .
$$

Next we multiply $(2.14)_{3}$ by a test function $\bar{\psi}$ and integrate over $\Gamma$ :

$$
\int_{\Gamma}\left(\frac{1}{2} w^{-}+D\left[w^{-}\right]-S[\varphi]\right) \bar{\psi} d s=\int_{\Gamma} u^{0} \bar{\psi} d s .
$$

We perform similar calculations for $\left(\mathrm{P}_{1}\right)$ and obtain:

$$
\begin{gathered}
-\int_{\Omega}\left(\mu \nabla w \cdot \nabla \bar{v}-\beta^{2} w \bar{v}\right) d x+\int_{\Gamma}\left(\frac{1}{2} \varphi+N[\varphi]\right) \bar{v}^{-} d s=-\int_{\Gamma} u_{n}^{0} \bar{v}^{-} d s \\
\int_{\Gamma}\left(w^{-}-S[\varphi]\right) \bar{\psi} d s=\int_{\Gamma} u^{0} \bar{\psi} d s .
\end{gathered}
$$

Eqs. (2.20) and (2.21) give our variational problems. Let us introduce some notation. We write $U, V$ for pairs $U=\{w, \varphi\}$ and $V=\{v, \psi\}$. For functions $\alpha, \beta$ defined on $\Gamma$ put $\langle\alpha, \beta\rangle=\int_{\Gamma} \alpha \beta$ ds. Now define the bilinear forms, $\mathscr{A}(U, V), \mathscr{B}(U, V)$ by

$$
\mathscr{A}(U, V)=A_{11}(w, v)+A_{12}(\varphi, v)+A_{21}(w, \psi)+A_{22}(\varphi, \psi),
$$

where

$$
\begin{aligned}
& A_{11}(w, v)=-\int_{\Omega}\left(\nabla w \cdot \nabla \bar{v}-\beta^{2} w \bar{v}\right) d x, \quad A_{12}(\varphi, v)=\langle\varphi, \bar{v}\rangle, \\
& A_{21}(w, \psi)=\left\langle\bar{\psi}, \frac{1}{2} w^{-} D\left[w^{-}\right]\right\rangle, \quad A_{22}(\varphi, \psi)=-\langle\bar{\psi}, S[\varphi]\rangle, \\
& \mathscr{B}(U, V)=B_{11}(w, v)+B_{12}(\varphi, v)+B_{21}(w, \psi)+B_{22}(\varphi, \psi) \text {, }
\end{aligned}
$$

where

$$
\begin{gathered}
B_{11}(w, v)=A_{11}(w, v), \quad B_{12}(\varphi, v)=\left\langle\frac{1}{2} \varphi+N[\varphi], \bar{v}^{-}\right\rangle, \\
B_{21}(w, \psi)=\left\langle\bar{\psi}, w^{-}\right\rangle, \quad B_{22}(\varphi, \psi)=-\langle\bar{\psi}, S[\varphi]\rangle .
\end{gathered}
$$


We also introduce the functionals,

$$
\mathscr{F}(\{v, \psi\})=\left\langle\bar{\psi}, u^{0}\right\rangle, \quad \mathscr{G}(\{v, \psi\})=\left\langle u_{n}^{0}, \bar{v}^{-}\right\rangle+\left\langle\bar{\psi}, u^{0}\right\rangle .
$$

Problem $\left(\mathrm{VP}_{0}\right)$. Find $U=\{w, \varphi\}$ such that for all $V=\{v, \psi\}, \mathscr{A}(U, \mathrm{~V})=\mathscr{F}(V)$.

$\operatorname{Problem}\left(\mathrm{VP}_{1}\right)$. Find $U=\{w, \varphi\}$ such that for all $V=\{v, \psi\}, B(U, V)=\mathscr{G}(V)$.

These problems will be analyzed in the subsequent sections. To anticipate, it will turn out the appropriate space on which to study them is the Hilbert space $\mathscr{H}=H_{1}(\Omega) \times$ $H_{-1 / 2}(\Gamma)$. Two observations are important. First, boundary conditions are natural. ${ }^{* *}$ This means that when we approximate, as below, with finite elements there are no boundary restrictions on those elements. Second, the problems are "adjoint" in the following sense:

$$
\mathscr{B}(U, V)=\mathscr{A}(\bar{V}, \bar{U}) \text {. }
$$

Let us verify this fact. We have by $(2.3)$ and (2.26) $B_{11}(w, v)=A_{11}(w, v)=A_{11}(\bar{v}, \bar{w}), B_{21}(w$, $\psi)=\left\langle\bar{\psi}, w^{-}\right\rangle=A_{12}(\bar{\psi}, \bar{w})$. From $(2.6)_{1}$ we have $B_{22}(\varphi, \psi)=-\langle\bar{\psi}, S[\varphi]\rangle=-\langle\varphi$, $S[\bar{\psi}]\rangle=A_{22}(\bar{\psi}, \bar{\varphi})$. Finally from $(2.6)_{2}$ we have $\langle\varphi, N[\psi]\rangle=\langle\psi, D[\varphi]\rangle$. Hence $B_{12}(\varphi$, $v)=\left\langle\frac{1}{2} \varphi+N[\varphi], \bar{v}^{-}\right\rangle=\left\langle\varphi, \frac{1}{2} \bar{v}^{-}+D\left[\bar{v}^{-}\right]\right\rangle=A_{21}(\bar{v}, \bar{\varphi})$.

We consider now approximate solutions of $\left(\mathrm{VP}_{0}\right)$ and $\left(\mathrm{VP}_{1}\right)$. We choose finitedimensional subspaces $S^{h}$ and $\Gamma^{h}$ of the spaces $H_{1}(\Omega)$ and $H_{-1 / 2}(\Gamma)$, put $\mathscr{H}^{h}=S^{h} x \Gamma^{h}$ and consider the approximate problems:

Problem $\left(\mathrm{AVP}_{0}\right):$ Find $U^{h} \in \mathscr{H}^{h}$ such that for all $V^{h} \in \mathscr{H}^{h}$,

$$
\mathscr{A}\left(U^{h}, V^{h}\right)=\mathscr{F}\left(V^{h}\right) \text {. }
$$

Problem $\left(\mathrm{AVP}_{1}\right): \quad$ Find $U^{h} \in \mathscr{H}^{h}$ such that for all $\mathrm{V}^{h} \in \mathscr{H}^{h}$

$$
\mathscr{B}\left(U^{h}, V^{h}\right)=\mathscr{G}\left(V^{h}\right) \text {. }
$$

Each of the approximate problems is equivalent to a set of algebraic equations. Suppose $v_{1}^{h}, \ldots v_{N h}^{h}$ is a (real) basis for $\mathscr{H}^{h}$. If we seek $U^{h}=\sum_{1}^{N^{h}} U_{k}^{h} v_{k}^{h}$ and define $U^{h}=$ $\left(U_{1}^{h}, \ldots U_{N^{h}}^{h}\right) \in \mathbb{R}^{N^{h}}$ then $(2.25)$ are equivalent to

$$
\mathscr{A} \mathrm{U}^{h}=\mathscr{F}^{h}, \quad \mathscr{B} \mathrm{U}^{h}=\mathscr{G}^{h}
$$

where

$$
(\mathscr{A})_{i j}=\mathscr{A}\left(v_{j}^{h}, v_{i}^{h}\right), \quad \mathscr{B}=\mathscr{A}^{t}, \quad\left(\mathscr{F}^{h}\right)_{i}=\mathscr{F}\left(v_{i}^{h}\right), \quad\left(\mathscr{G}^{h}\right)_{i}=\mathscr{G}\left(v_{i}^{h}\right) .
$$

Let us study the structure of Eqs. (2.29). Let us suppose that our basis for $S^{h}$ has the form $\omega_{1}^{h}, \ldots \omega_{N_{\mathbf{n}^{h}}^{h}}^{h} \gamma_{1}^{h}, \ldots \gamma_{N_{\Gamma^{h}}}^{h}$ with $\omega_{j}^{h} \equiv 0$ on $\Gamma$ and let $\sigma_{1}^{h}, \ldots \sigma_{m}^{h}$ be a basis for $\Gamma^{h}$. Then $\mathbf{U}^{h}$ has a corresponding decomposition into $\mathbf{w}_{\Omega}^{h}, \mathbf{w}_{\Gamma}^{h}, \phi^{h}$ and (2.29) assumes the form

$$
\begin{aligned}
& \left(\begin{array}{ccc}
\mathbf{A}_{\Omega \Omega}^{h} & \mathbf{A}_{\Omega \Gamma}^{h} & \mathbf{0} \\
\mathbf{A}_{\Gamma \Omega}^{h} & \mathbf{A}_{\Gamma \Gamma}^{h} & \mathbf{A}_{\Gamma \Phi}^{h} \\
\mathbf{0} & \mathbf{A}_{\Phi \Gamma}^{h} & \mathbf{A}_{\Phi \Phi}^{h}
\end{array}\right)\left(\begin{array}{c}
\mathbf{w}_{\Omega}^{h} \\
\mathbf{w}_{\Gamma}^{h} \\
\boldsymbol{\phi}^{h}
\end{array}\right)=\left(\begin{array}{c}
\mathbf{0} \\
\mathscr{F}_{\Gamma}^{h} \\
\mathscr{F}_{\Phi}^{h}
\end{array}\right) \\
& \left(\begin{array}{ccc}
\mathbf{B}_{\Omega \Omega}^{h} & \mathbf{B}_{\Omega \Gamma}^{h} & \mathbf{0} \\
\mathbf{B}_{\Gamma \Omega}^{h} & \mathbf{B}_{\Gamma \Gamma}^{h} & \mathbf{B}_{\Gamma \Phi}^{h} \\
\mathbf{0} & \mathbf{B}_{\Phi \Gamma}^{h} & \mathbf{B}_{\Phi \Phi}^{h}
\end{array}\right)\left(\begin{array}{c}
\mathbf{w}_{h}^{\Omega} \\
\mathbf{w}_{\Gamma}^{h} \\
\boldsymbol{\phi}^{h}
\end{array}\right)=\left(\begin{array}{c}
\mathbf{0} \\
\mathscr{G}_{\Gamma}^{h} \\
\mathscr{G}_{\Phi}^{h}
\end{array}\right) .
\end{aligned}
$$

** The variational principles here are similar in this respect to those of $[10]$ for boundary-value problems. 
Here we have

$$
\begin{gathered}
\left(\mathbf{A}_{\Omega \Omega}^{h}\right)_{i j}=\left(\mathbf{B}_{\Omega \Omega}^{h}\right)_{i j}=\mathbf{A}_{11}\left(\omega_{j}, \omega_{i}\right), \quad i, j=1, \ldots N_{\Omega}^{h}, \\
\left(\mathbf{A}_{\Omega \Gamma}^{h}\right)_{i j}=\left(\mathbf{B}_{\Omega \Gamma}^{h}\right)_{i j}=A_{11}\left(\gamma_{j}, \omega_{i}\right), \quad i=1, \ldots N_{\Omega}^{h}, \quad j=1, \ldots N_{\Gamma}^{h}, \\
\left(\mathbf{A}_{\Gamma \Gamma}^{h}\right)_{i j}=\left(\mathbf{B}_{\Gamma \Gamma}^{h}\right)_{i j}=A_{11}\left(\gamma_{j}, \gamma_{i}\right), \quad i, j=1, \ldots N_{\Gamma}^{h}, \\
\left(\mathbf{A}_{\Gamma \Phi}^{h}\right)_{i j}=A_{12}\left(\sigma_{j}, \gamma_{i}\right), \quad i=1, \ldots N_{\Gamma}^{h}, \quad j=1, \ldots M ; \quad \mathbf{B}_{\Phi \Gamma}^{h}=\left(\mathbf{A}_{\Gamma \Phi}^{h}\right)^{t}, \\
\left(\mathbf{A}_{\Phi \Gamma}^{h}\right)_{i j}=A_{21}\left(\gamma_{j}, \sigma_{i}\right), \quad i=1, \ldots M, \quad j=1, \ldots N_{\Gamma}^{h} ; \quad \mathbf{B}_{\Gamma \Phi}^{h}=\left(\mathbf{A}_{\Phi \Gamma}^{h}\right)^{t}, \\
\left(\mathbf{A}_{\Phi \Phi}\right)_{i j}=\left(\mathbf{B}_{\Phi \Phi}\right)_{i j}=A_{22}\left(\sigma_{j}, \sigma_{i}\right), \quad i, j=1, \ldots M .
\end{gathered}
$$

We point out that the form of Eqs. (2.31) permits condensation. Suppose we are solving $\mathrm{VP}_{0}$ (that is $\mathrm{P}_{0}$ ) and we are primarily concerned with the exterior region. Then we may eliminate $\mathbf{w}_{\boldsymbol{\Omega}}^{h}$ and consider the system:

$$
\left(\begin{array}{ll}
\tilde{\mathbf{A}}_{\Gamma \Gamma}^{h} & \mathbf{A}_{\Gamma \Phi}^{h} \\
\mathbf{A}_{\Phi \Gamma}^{h} & \mathbf{A}_{\Phi \Phi}^{h}
\end{array}\right)\left(\begin{array}{l}
\mathbf{w}_{\Gamma}^{h} \\
\phi^{h}
\end{array}\right)=\left(\begin{array}{c}
\mathscr{F}_{\Gamma}^{h} \\
\mathscr{F}_{\Phi}^{h}
\end{array}\right)
$$

where $\tilde{\mathbf{A}}_{\Gamma \Gamma}^{h}=\mathbf{A}_{\Gamma \Gamma}^{h}-\mathbf{A}_{\Gamma \Omega}^{h}\left(A_{\Omega \Omega}^{h}\right)^{-1} \mathbf{A}_{\Omega \Gamma}^{h}$. Other combinations are clearly possible, also for $\left(\mathrm{VP}_{1}\right)$.

Remark: We observe that other auxiliary problems are possible. Let us keep $\phi$ as in (2.15) but now let $\lambda=\mu w_{n}^{-}$so that we have two boundary functions. We multiply (VP $\left.\mathrm{P}_{0}\right)$ by $(1-\alpha)$ and $\left(\mathrm{VP}_{1}\right)$ by $\alpha$ and add the results. If we put $\mathscr{U}=(w, \lambda, \phi)$ and $\mathscr{V}=(v, \chi, \psi)$ and define $\mathscr{A}_{\alpha}(\mathscr{U}, \mathscr{V}), \mathscr{F}_{\alpha}(\mathscr{V})$ by

$$
\begin{aligned}
\mathscr{A}_{\alpha}(\mathscr{U}, \mathscr{V})= & (1-\alpha) A_{11}(w, v)+(1-\alpha) A_{12}(\lambda, v)+\alpha B_{12}(\phi, v) \\
& +\alpha B_{11}(w, v)+\alpha B_{21}(w, \chi)+\alpha B_{22}(\phi, \chi) \\
& +(1-\alpha) A_{21}(w, \psi)+(1-\alpha) A_{22}(\lambda, \psi), \\
\mathscr{F}^{\alpha}(\mathscr{V})= & \alpha\left\langle u_{n}^{0}, \bar{v}^{-}\right\rangle+\alpha\left\langle\bar{x}, u^{0}\right\rangle+(1-\alpha)\left\langle\bar{\psi}, u^{0}\right\rangle
\end{aligned}
$$

then we obtain:

Problem $\left(\mathrm{VP}_{\alpha}\right) . \quad$ Find $\mathscr{U}=\{w, \lambda, \varphi\}$ such that for all $\mathscr{V}=\{v, x, \psi\}$,

$$
\mathscr{A}_{\alpha}(\mathscr{U}, \mathscr{V})=\mathscr{F}_{\alpha}(v) \text {. }
$$

Observe that for $\alpha=0\left(\mathrm{VP}_{\alpha}\right)$ becomes $\left(\mathrm{VP}_{0}\right)$ and for $\alpha=1$ it becomes $\left(\mathrm{VP}_{1}\right)$. Further, when $\alpha=\frac{1}{2}$ we have, by (2.27),

$$
\begin{gathered}
\frac{1}{2} B_{21}(w, \chi)=\frac{1}{2} A_{12}(\bar{\chi}, \bar{w}), \quad \frac{1}{2} A_{21}(w, \psi)=\frac{1}{2} B_{12}(\bar{\psi}, \bar{w}), \\
\frac{1}{2} A_{22}(\lambda, \psi)=\frac{1}{2} B_{22}(\bar{\psi}, \bar{\lambda}),
\end{gathered}
$$

so that $\mathscr{A}_{\alpha}(\mathscr{U}, \mathscr{V})=\mathscr{A}_{\alpha}(\overline{\mathscr{V}}, \overline{\mathscr{U}})$. In the finite-dimensional approximation this means that the matrices will be symmetric if we use real basis elements. The price we pay for this symmetry is that the system (2.31) will be replaced by a set of $N_{\Omega}^{h}+N_{\Gamma}^{h}+2 M$ equations instead of $N_{\Omega}^{h}+N_{\Gamma}^{h}+M$. The form will be, for $\alpha=\frac{1}{2}$,

$$
\left(\begin{array}{cccc}
\mathbf{A}_{\Omega \Omega}^{h} & \mathbf{A}_{\Omega \Gamma}^{h} & \mathbf{0} & \mathbf{0} \\
\left(\mathbf{A}_{\Omega \Gamma}^{h}\right)^{t} & \mathbf{A}_{\Gamma \Gamma}^{h} & \frac{1}{2} \mathbf{A}_{\Gamma \Phi}^{h} & \frac{1}{2}\left(\mathbf{A}_{\Phi \Gamma}^{h}\right)^{t} \\
\mathbf{0} & \frac{1}{2}\left(\mathbf{A}_{\Gamma \Phi}^{h}\right)^{t} & \mathbf{0} & \frac{1}{2} \mathbf{A}_{\Phi \Phi}^{h} \\
\mathbf{0} & \frac{1}{2} \mathbf{A}_{\Phi \Gamma}^{h} & \frac{1}{2} \mathbf{A}_{\Phi \Phi}^{h} & \mathbf{0}
\end{array}\right)\left(\begin{array}{c}
\mathbf{w}_{\Omega}^{h} \\
\mathbf{w}_{\Gamma}^{h} \\
\lambda^{h} \\
\boldsymbol{\phi}^{h}
\end{array}\right)=\left(\begin{array}{c}
0 \\
\frac{1}{2} \mathscr{F}_{\Gamma}^{h} \\
\frac{1}{2} \mathscr{F}_{\Phi}^{h} \\
\frac{1}{2} \mathscr{F}_{\Phi}^{h}
\end{array}\right)
$$

where the $A^{h}$ 's and $\mathscr{F}^{h}$ 's are as in $(2.31)_{1}$. 
The variational problem $\left(\mathrm{VP}_{\alpha}\right)$ is equivalent to the boundary problem:

$\operatorname{Problem}\left(\mathrm{P}_{\alpha}\right)$ : Find $w, \lambda$, $\phi$ such that

$$
\begin{gathered}
L_{\mu} w+\beta^{2} w=0 \quad \text { in } \quad \Omega, \quad-\mu w_{n}^{-}+(1-\alpha) \lambda+\alpha\left(\frac{1}{2} \phi+N[\phi]=-\alpha u_{n}^{0},\right. \\
\alpha w^{-} \alpha S[\phi]=\alpha u^{0}, \quad(1-\alpha) \frac{1}{2} w^{-}+(1-\alpha) D\left[w^{-}\right]-(1-\alpha) S[\lambda]=(1-\alpha) u^{0} .
\end{gathered}
$$

This problem can be analyzed in the same way as the special cases $\left(P_{0}\right)$ and $\left(P_{1}\right)$.

3. Existence theorems. We will seek generalized solutions of our problems. For these we need the Sobolev spaces $H_{r}(\Omega), r \geq-1$ and $H_{s}(\Gamma), s \geq-\frac{1}{2}$ for complex-valued functions. We put

$$
H_{r}^{\text {loc }}\left(\Omega^{+}\right)=\left\{w: w \in H_{r}\left(\Omega_{R}^{+}\right) \text {for any } R>0, \Omega_{R}^{+}=\Omega^{+} \cap(|x|<R)\right\} .
$$

For any $\varepsilon \geq 0$ we put

$$
\begin{gathered}
\mathscr{H}_{\varepsilon}=H_{1+\varepsilon}(\Omega) \times H_{-1 / 2+\varepsilon}(\Gamma), \\
\|U U\|_{\varepsilon}^{2}=\|\|\{w, \phi\}\left\|_{\varepsilon}^{2}=\right\| w\left\|_{1+\varepsilon}^{2}(\Omega)+\right\| \phi \|_{-1 / 2+\varepsilon}^{2}(\Omega) \text { for } U \in \mathscr{H}_{\varepsilon}(e) .
\end{gathered}
$$

We write $\langle\phi, \chi\rangle$ for the pairing of $\phi \in H_{-s}(\Gamma)$ with $\chi \in H_{s}(\Gamma)$.

We recall the trace theorems. If $w \in H_{r}(\Omega)\left(H_{r}^{\text {loc }}\left(\Omega^{+}\right)\right)$then if $r \geq 1 w$ has a trace $w^{-}\left(w^{+}\right)$in $H_{r-1 / 2}(\Gamma)$ and if $r \geq \frac{3}{2}$ then $w$ has a (trace) normal derivative $w_{n}^{-}\left(w_{n}^{+}\right) \in$ $H_{r-3 / 2}(\Gamma)$. If $w$ in addition satisfies a homogeneous second-order elliptic equation in $\Omega$ $\left(\Omega^{+}\right)$then $w_{n}^{-}\left(w_{n}^{+}\right)$is in $H_{r-3 / 2}(\Gamma)$ for any $r \geq 1$.

The operators $\mathscr{S}$ and $\mathscr{D}$ of Sec. 2 extend to $\phi$ 's in $H_{s}(\Gamma)$ for $s \geq-\frac{1}{2}$. One has only to replace the integrals by brackets, for instance $\mathscr{S}[\phi](x)=\langle\phi, g(x, \cdot)\rangle$. These extended operators will still satisfy (2.1) in $\Omega$ and $\Omega^{+}$as well as (1.13). The operators $S, N$, and $D$ also extend and all the boundary limit results of Sec. 2 hold when translated in trace statements. These ideas are discussed in [8]. We summarize the results.

Lemma 3.1. For any $s \geq-\frac{1}{2}, S$ is a bounded map $H_{s}(\Gamma) \rightarrow H_{s+1}(\Gamma)$. $N$ and $D$ are bounded maps $H_{s}(\Gamma) \rightarrow H_{s+2}(\Gamma)$.

LemMA 3.2 (i). If $\phi \in H_{s}(\Gamma), s \geq-\frac{1}{2}, \mathscr{S}[\phi] \in H_{s+3 / 2}(\Omega) \cap H_{s+3 / 2}^{\text {loc }}\left(\Omega^{+}\right)$and $\mathscr{S}[\phi]^{ \pm}=$ $S[\phi],[\partial \mathscr{S} / \partial n]^{ \pm}= \pm \frac{1}{2} \phi+N[\phi]$.

(ii). If $\phi \in H_{s}(\Gamma), s \geq \frac{1}{2}, \mathscr{D}[\phi] \in H_{s+1 / 2}(\Omega) \cap H_{s+1 / 2}^{\text {loc }}\left(\Omega^{+}\right)$and $(\mathscr{D}[\phi])^{ \pm}=\mp \frac{1}{2} \phi+$ $D[\phi]$ and $(\partial \mathscr{D}[\phi] / \partial n)^{ \pm} \in H_{s-1}(\Gamma)$.

There is another result which generalizes Lemma 2.1 (ii) and can be obtained from the ideas in [8].

Lemma 3.3. For any $s \geq-\frac{1}{2}$ the map $S: H_{s}(\Gamma) \rightarrow H_{s+1}(\Gamma)$ is surjective.

We can use the above ideas to give generalized versions of the problems in Sec. 2. At the same time we will introduce some further inhomogeneities for use in the next section. We introduce a little more notation. The data in our generalized problems will be in the space $\mathscr{W}_{\varepsilon}=H_{-1+\varepsilon}(\Omega) \times H_{-1 / 2+\varepsilon}(\Gamma) \times H_{1 / 2+\varepsilon}(\Gamma)$ and for $(F, p, q) \in \mathscr{W}_{\varepsilon}$ we set

$$
\text { III }(F, p, q)\|\|_{\varepsilon}^{2}=\|F\|_{-1+\varepsilon}^{2}(\Omega)+\|p\|_{-1 / 2+\varepsilon}^{2}(\Gamma)+\|q\|_{1 / 2+\varepsilon}^{2}(\Gamma) .
$$

$\operatorname{Problem}\left(\widetilde{\mathrm{P}}_{0}\right)$. Given $(F, p, q) \in \mathscr{W}_{\varepsilon}$ find $(w, \phi) \in \mathscr{H}_{\varepsilon}$ such that

$$
L_{\mu} w+\beta^{2} w=F \quad \text { in } \quad \Omega, \quad \mu w_{n}^{-}-\phi=p, \quad \frac{1}{2} w^{-}+D\left[w^{-}\right]-S[\phi]=q .
$$

$\operatorname{Problem}\left(\tilde{\mathrm{P}}_{1}\right)$. Given $(F, p, q) \in \mathscr{W}_{\varepsilon}$ find $(w, \phi) \in \mathscr{H}_{\varepsilon}$ such that

$$
L_{\mu} w+\beta^{2} w=F \quad \text { in } \quad \Omega, \quad \mu w_{n}^{-}-\frac{1}{2} \phi-N[\phi]=p, \quad w^{-}-S[\phi]=q .
$$


The equation in $\Omega$ is to be interpreted in the distribution sense. $w$ is to have a trace normal derivative in $H_{-1 / 2+\varepsilon}(\Gamma)$ and the boundary equations are to be interpreted as in the lemmas.

We will establish the following results.

TheOREM 3.1. Problems $\left(\tilde{\mathrm{P}}_{0}\right)$ and $\left(\widetilde{\mathrm{P}}_{1}\right)$ have unique solutions $(w, \varphi)$. Moreover, there exists a constant $M>0$ such that for both solutions

$$
\||(w, \varphi)|\|_{\varepsilon} \leq M\left|\|(F, p, q) \mid\|_{\varepsilon} .\right.
$$

Corollary 3.1. If $u^{0} \in H_{k}\left(x_{2} \geq 0\right)$ for $k>3$ then (P) has a solution.

Proof of Corollary. Let $F=0, p=u_{n}^{0}, q=u^{0}$ on $\Gamma$. $u^{0} \in H_{k}\left(x_{2} \geq 0\right)$ implies $p \epsilon$ $H_{-1 / 2-(k-1)}(\Gamma)$ and $q \in H_{1 / 2-(k-1)}(\Gamma)$. Then $\left(\tilde{\mathrm{P}}_{0}\right)$ has a solution $(w, \varphi) \in \mathscr{H}_{k-1}$, which means $w \in \mathscr{H}_{k}(\Omega), \varphi \in \mathscr{H}_{k-3 / 2}(\Gamma)$. Since $k>3$ this means $w \in K(\Omega)$ and $\varphi \in C(\Gamma)$. It follows that $(w, \varphi)$ is a solution of (2.14) pointwise and by Theorem 2.1(ii) we obtain a solution of $(\mathrm{P})$. A similar proof can be given using $\left(\widetilde{\mathrm{P}}_{1}\right)$.

Let us discuss the variational problems. The weakest solutions $(w, \varphi)$ will be in $\mathscr{H}_{0}=$ $H_{1}(\Omega) \times H_{-1 / 2}(\Gamma)$ and these will correspond to $(F, p, q) \in \mathscr{W}_{0}$. If we consider the bilinear forms $\mathscr{A}(U, V)$ and $\mathscr{B}(U, V)$ of Sec. 2 we see that they are meaningful for $(U, V) \in \mathscr{H}_{0}$ $\times \mathscr{H}_{0}$, provided that we interpret the brackets as pairings. One checks that indeed $A$ and $\mathscr{B}$ are bounded forms on $\mathscr{H}_{0} \times \mathscr{H}_{0}$,

$$
|\mathscr{A}(U, V)| \leq \bar{A}|||U|\left\|_{0}\left|\|V\|_{0}, \quad\right| \mathscr{B}(U, V)|\leq \bar{B}||U|\right\|_{0}\||| V\|_{0},
$$

for some constants $\bar{A}$ and $\bar{B}$. We can extend the functionals $\mathscr{F}$ and $\mathscr{G}$ to

$$
\mathscr{F}(V)=\mathscr{F}(\{v, \psi\}) \equiv \mathscr{G}(V) \equiv \mathscr{G}(\{v, \psi\})=\langle F, \bar{v}\rangle+\left\langle p, \bar{v}^{-}\right\rangle+\langle\bar{\psi}, q\rangle .
$$

The right side of (3.8) is meaningful for $(F, p, q) \in \mathscr{W}_{0}$ if $\langle F, \bar{v}\rangle$ is interpreted as the pairing of $F \in H_{-1}(\Omega)$ and $\bar{v} \in H_{1}(\Omega)$. Moreover, they are bounded functionals:

$$
|\mathscr{F}(V)|=|\mathscr{G}(V)| \leq \bar{F}\left\|\left|V\left\|_{0}\right\|(F, p, q)\right|\right\|_{0} .
$$

We have then the generalized variational problems:

$\operatorname{Problem}\left(\mathrm{VP} \widetilde{\mathrm{P}}_{0}\right)\left(\left(\mathrm{VP} \widetilde{\mathrm{P}}_{1}\right)\right)$. Find $U \in \mathscr{H}_{0}$ such that for all $V \in \mathscr{H}_{0}$,

$$
\mathscr{A}(U, V)=\mathscr{F}(V) \quad(\mathscr{B}(U, V)=\mathscr{G}(V) .
$$

One checks that $\left(V \widetilde{\mathrm{P}}_{0}\right)\left(\left(\mathrm{V} \widetilde{\mathrm{P}}_{1}\right)\right)$ have unique solutions if and only if $\left(\widetilde{\mathrm{P}}_{0}\right),\left(\widetilde{\mathrm{P}}_{1}\right)$ have unique solutions $U \in \mathscr{H}_{0}$. Hence Theorem 3.1 will guarantee that $\left(\mathrm{VP} \widetilde{P}_{0}\right)$ and $\left(\mathrm{VP}_{1}\right)$ have unique solutions.

We turn now to the proof of Theorem 3.1. The idea is to reduce the problems to Riesz-Schauder equations on $H_{s}(\Gamma)$. To do this we first study the boundary-value problem: given $G$ and $P$, find $v$ such that

$$
L_{\mu} v+\beta^{2} v=G \text { in } \Omega, \quad \mu w_{n}^{-}=P \text { on } \Gamma .
$$

Under hypothesis $\left(\mathrm{H}_{2}\right)$ it is known that this problem has a unique solution of the following form (see [11]):

$$
v=\mathscr{I}_{1}[G]+\mathscr{I}_{2}[P], \quad v^{-}=\mathscr{I}_{1}^{-}[G]+\mathscr{I}_{2}^{-}[P]
$$

where

$$
\begin{array}{rr}
\mathscr{I}_{1}: H_{-1+\varepsilon}(\Omega) \rightarrow H_{1+\varepsilon}(\Omega), & \mathscr{I}_{2}: H_{-1 / 2+\varepsilon}(\Omega) \rightarrow H_{1+\varepsilon}(\Omega) . \\
\mathscr{I}_{1}^{-}: H_{-1+\varepsilon}(\Omega) \rightarrow H_{1 / 2+\varepsilon}(\Gamma), & \mathscr{I}_{2}^{-}: H_{-1 / 2+\varepsilon}(\Gamma) \rightarrow H_{1 / 2+\varepsilon}(\Gamma)
\end{array}
$$

are all bounded maps. 
We need the following refinement of this result.

Lemma 3.4. $\mathscr{I}_{2}[P]=-2 \mathscr{S}[P / \mu]+R[P], \mathscr{I}_{2}^{-}[P]=-2 S[P / \mu]+R^{-}[P]$, where $R[P]$ : $H_{-1 / 2+\varepsilon}(\Gamma) \rightarrow H_{2+\varepsilon}(\Omega), R^{-}[P]: H_{-1 / 2+\varepsilon}(\Gamma) \rightarrow H_{3 / 2+\varepsilon}(\Gamma)$ are bounded.

Proof. Let us put $\mathscr{I}_{1}[P]=-2 \mathscr{S}[P / \mu]+w$. By (2.5) and (3.8) we have

$$
\mu w_{n}^{-}=2 \mu N\left[\frac{P}{\mu}\right] \equiv n[P] \text {. }
$$

Also

$$
L_{\mu} w+\beta^{2} w=2 \nabla \mu \cdot \nabla \mathscr{S}\left[\frac{P}{\mu}\right]+\left(\beta^{2}-2 \mu\right) \mathscr{S}\left[\frac{P}{\mu}\right] \equiv J[P] .
$$

Hence $w=\mathscr{I}_{1}[J[P]]+\mathscr{I}_{2}[n[P]]$.

Now by Lemma (3.2) $P \in H_{-1 / 2+\varepsilon}(\Gamma)$ implies $\mathscr{P}[P / \mu] \in H_{1+\varepsilon}(\Omega)$ so that $J[P] \in H_{\varepsilon}(\Omega)$. Also Lemma (3.1) implies $n \in H_{3 / 2+\varepsilon}(\Gamma)$. Thus $\mathscr{I}_{1}[J[P]] \in H_{2+\varepsilon}(\Omega), \mathscr{I}_{2}[n[P]] \in H_{3+2}(\varepsilon)$. This and the trace theorem yield the conclusion.

We now prove Theorem 3.1 for $\left(\tilde{P}_{0}\right)$. We seek the solution of (3.4) in the form

$$
w=\mathscr{I}_{1}(F)+\mathscr{I}_{2}[P]
$$

where $P$ is the (unknown) value of $\mu w_{n}^{-} .(3.4)_{2}$ yields $\varphi=P-p$. We substitute this result and the result of Lemma (3.4) into (3.4) $)_{3}$ to obtain:

$$
\begin{aligned}
-S\left[\frac{P}{\mu}\right]+R^{-}[P]+D\left\{-2 S\left[\frac{P}{\mu}\right]+R^{-}[P]\right\} & -S[P]+S[p] \\
& =q-\frac{1}{2} \mathscr{I}_{1}^{-}[F]-D \mathscr{I}_{1}^{-}[F] .
\end{aligned}
$$

Applying $S^{-1}$ to this equation we obtain

$$
P+\mathscr{M}[P]=P_{0}
$$

where

$$
\begin{aligned}
\mathscr{M}[P] & =\left(\frac{1}{\mu}+1\right)^{-1} S^{-1}\left\{\frac{1}{2} R^{-}[P]-2 D S\left[\frac{P}{\mu}\right]+D R^{-}[P]\right\}, \\
P_{0} & =\left(\frac{1}{\mu}+1\right)^{-1}\left\{p-S^{-1}\left[q-\frac{1}{2} \mathscr{I}_{1}^{-}[F]-D \mathscr{I}_{1}^{-}[F]\right]\right\} .
\end{aligned}
$$

If we take account of the mapping properties developed we find that $\mathscr{M}$ maps $H_{s}(\Gamma)$ into $H_{s+1}(\Gamma)$ and hence is compact as a map on $H_{s}(\Gamma)$. We have $p \in H_{-1 / 2+\varepsilon}(\Gamma)$ and $q \in H_{1 / 2+\varepsilon}(\Gamma)$ so that $P_{0} \in H_{-1 / 2+\varepsilon}(\Gamma)$. Thus (3.19) is a Riesz-Schauder equation on $H_{-1 / 2+\varepsilon}(\Gamma)$. If we can establish that the only solution of the homogeneous equation

$$
P+\mathscr{M}[P]=0
$$

is $P \equiv 0$ it will follow that (3.18) has a unique solution $P \in H_{-1 / 2+\varepsilon}(\Gamma)$ for any $P_{0} \in$ $H_{-1 / 2+\varepsilon}(\Gamma)$. Reversing the steps it is then easy to verify that (3.16) yields a solution of $\left(\tilde{\mathrm{P}}_{0}\right)$.

Suppose $P \in H_{-1 / 2+\varepsilon}(\Gamma)$ is a solution of (3.20). Then since $\mathscr{M}$ maps $H_{0}(\Gamma)$ into $H_{s+1}(\Gamma)$ it follows that $P \in H_{s}(\Gamma)$ for any $s$. Forming (3.16) with $F=0$ we then obtain a solution of $\left(\tilde{\mathrm{P}}_{0}\right)$ in $\mathscr{H}_{\varepsilon}(\Omega)$ for any $\varepsilon$. By Theorem 2.1 this yields a solution of $(P)$ with $u^{0} \equiv 0$, which is identically zero by Theorem 1.1 . But then $P=\mu w_{n}^{-} \equiv 0$. We conclude that (3.18) always has a solution. This completes the existence portion of Theorem 3.1 and the argument just given also establishes the uniqueness. 
The proof for $\left(\mathrm{P}_{1}\right)$ is almost the same. We again seek $w$ in the form (3.16) with $P$ unknown. Then (3.5) ${ }_{3}$ and Lemma 3.4 yield

$$
-2 S\left[\frac{P}{\mu}\right]+R^{-}\left[\frac{P}{\mu}\right]-S[\varphi]=q
$$

or

$$
\varphi=-\frac{2 P}{\mu}-S^{-1}[q]+S^{-1} R^{-}\left[\frac{P}{\mu}\right] .
$$

We substitute into $(3.5)_{2}$ and obtain another equation of the form (3.18) which can be analyzed in the same way.

4. Convergence of the approximate problems. In this section we describe a situation in which we can guarantee that the finite-dimensional problems $\left(\mathrm{AV} \widetilde{P}_{0}\right)$ and $\left(\mathrm{AVP} \widetilde{P}_{1}\right)$ have unique solutions $U^{h} \in \mathscr{H}^{h}$ for each value of the parameter $h$ and that $U^{h}$ tends to the solutions of $\left(\widetilde{\mathrm{P}}_{0}\right)$ and $\left(\widetilde{\mathrm{P}}_{1}\right)$ as $h \rightarrow 0$. The setting is a familiar one from [5] and [10] and other similar work. We assume that $S^{h_{\Omega}}$ is a finite-element approximation space for $H_{1}(\Omega)$; that is, $h_{\Omega}$ is a mesh parameter and the functions in $S^{h_{\Omega}}$ are piecewise polynomials. Similarly, $\Gamma^{h_{\Gamma}}$ will be a finite element approximation space for $H_{-1 / 2}(\Gamma)$. We need the following properties.

Approximation properties:

(A.1) There exists a constant $\gamma_{1}>0$ and an integer $k>1$ such that for any $w \in H_{l}(\Omega)$, $1 \leq l \leq k$ there is a $w^{h_{\Omega}} \in S^{h_{\Omega}}$ with

$$
\left\|w-w^{h_{\Omega}}\right\|_{r}(\Omega) \leq \gamma_{1}\left(h_{\Omega}\right)^{l-r}\|w\|_{l}(\Omega), \quad 0 \leq r<l .
$$

(A.2) There exists a constant $\gamma_{2}>0$ and an integer $k^{\prime}>-\frac{1}{2}$ such that for any $\phi \in H_{l}$, (Г), $-\frac{1}{2} \leq l^{\prime} \leq k^{\prime}$ there is a $\phi^{h_{\Gamma}} \in S^{h_{\Gamma}}$ with

$$
\left\|\phi-\phi^{h_{\Gamma}}\right\|_{s}(\Gamma) \leq \gamma_{2}\left(h_{\Gamma}\right)^{l-s}\|\phi\|_{l}(\Gamma), \quad-\frac{1}{2} \leq s \leq l .
$$

For examples of spaces satisfying (A.1) and (A.2) we refer to [5] and [10]. We will define $h$ as

$$
h=h_{\Omega}+h_{\Gamma} .
$$

We assume (A.1) and (A.2) hold and we prove the following.

THEOREM 4.1. There exists a constant $\bar{h}>0$ such that if $h_{\Gamma}<\bar{h}$ then:

(i) $\left(\operatorname{AV} \tilde{P}_{0}\right)$ and $\left(\operatorname{AVP} \widetilde{P}_{1}\right)$ have unique solutions $U^{h}$.

(ii) If $U$ is the solution of $\left(\mathrm{VP}_{0}\right),\left(\left(V \tilde{\mathrm{P}}_{1}\right)\right)$ and hence of $\left(\widetilde{\mathrm{P}}_{0}\right)\left(\left(\tilde{\mathrm{P}}_{1}\right)\right)$, and $U=\{w, \phi\} \in$ $\mathscr{H}_{\varepsilon}(\Omega), \varepsilon<\min \left(k, k^{\prime}\right)$, then for any $\varepsilon^{\prime}<\varepsilon$ there is a constant $C$ such that

$$
\text { || } U-U^{h}\|\|_{\varepsilon^{\prime}} \leq C h^{\varepsilon-\varepsilon^{\prime}} .
$$

The key to proving Theorem 4.1 is to establish a coercivity inequality of the following form. We want to know that there is a constant $m>0$ such that for any $U^{h} \in \mathscr{H}^{h}$ we have

$$
\sup _{\substack{V^{h} \in \mathscr{P} \mathscr{P}^{h} \\ V^{h \neq 0}}}\left|\mathscr{A}\left(U^{h}, V^{h}\right)\right| \geq m\left\||| U^{h}\left|\left\|_{0}\right\|\right| V^{h}\right\|_{0},
$$


These inequalities will be proved later, but let us first show that they yield the proofs of Theorem 4.1.

Suppose that Eq. (2.29) for $\mathscr{F}^{h}=0$ has a solution $\mathbf{U}^{h}$. Form $U^{h}=\sum U_{j}^{h} V_{j}^{h}$. Then we have $\mathscr{A}\left(U^{h}, V^{h}\right)=0$ for all $V^{h} \in \mathscr{H}^{h}$. But then (4.3) implies that $U^{h} \equiv 0$; hence $\mathbf{U}^{h}=\mathbf{0}$. It follows that $(2.29)_{1}$ has a unique solution for any $\mathscr{F}^{h}$. Similarly $(2.29)_{2}$ has a unique solution.

To establish the convergence let $U$ and $U^{h}$ be solutions of $\left(\mathrm{V} \tilde{\mathrm{P}}_{0}\right)$ and $\left(\mathrm{AV} \widetilde{\mathrm{P}}_{0}\right)$ and put $E=U-W^{h}, \mathscr{E}=U^{h}-W^{h}$ where $W^{h}$ is an arbitrary element of $\mathscr{H}^{h}$. Then we have $\mathscr{A}\left(E-\mathscr{E}, V^{h}\right)=\mathscr{A}\left(U, V^{h}\right)-\mathscr{A}\left(U^{h}, V^{h}\right)=\mathscr{F}\left(V^{h}\right)-\mathscr{F}\left(V^{h}\right)=0$ for all $V^{h} \in \mathscr{H}^{h}$. Hence $\mathscr{A}\left(\mathscr{E}, V^{h}\right)=\mathscr{A}\left(E, V^{h}\right)$. If we apply (4.3) ${ }_{1}$ and (3.7) we obtain

$$
m\left|\|\mathscr{E}\| l_{0}\right|\left\|V^{h}\right\|_{0} \leq \sup _{V^{h} \in \mathscr{H}^{h}}\left|\mathscr{A}\left(\mathscr{E}, V^{h}\right)\right| \leq \sup _{V^{h} \in \mathscr{P}^{h}}\left|\mathscr{A}\left(E, V^{h}\right)\right| \leq \bar{A}\|E\|\left\|_{0}\right\| \mid V^{h} \|_{0}
$$

or $\|\mathscr{E} i\|_{0} \leq k\|\| E \|_{0}$ for some $k$ and

$$
\|\| U-U^{h} \mid\left\|_{0} \leq\right\| E-\mathscr{E}\left\|_{0} \leq(1+k)\right\| E\|\|_{0} \leq(1+k) \sup _{W^{h} \in \mathscr{P}^{h}}\left\|U-W^{h}\right\|_{0}
$$

Inequality (4.4) is an optimality result. It states that $U^{h}$ approximates $U$ in $\mathscr{H}^{h}$ as well as the best approximator. In particular, we can pick $W^{h}=\left\{w^{h_{\Omega}}, \phi^{h_{\Gamma}}\right\}$ as in (A.1) and (A.3). Suppose $U \in \mathscr{H}_{\varepsilon}$, that is, $w \in H_{1+\varepsilon}(\Omega), \phi \in H_{-1 / 2+\varepsilon}(\Gamma)$. Then we can take $l=1+\varepsilon$, $r=1+\varepsilon^{\prime}$ in (A.1) and $l=-\frac{1}{2}+\varepsilon, r=-\frac{1}{2}+\varepsilon^{\prime}$ in (A.2) and obtain

$$
\begin{gathered}
\left\|w-w^{h_{\Omega}}\right\|\left\|_{1+\varepsilon^{\prime}}(\Omega) \leq \gamma_{1}\left(h_{\Omega}\right)^{\varepsilon-\varepsilon^{\prime}}\right\| w \|_{1+\varepsilon}(\Omega), \\
\left\|\phi-\phi^{h_{\Gamma}}\right\|_{-1 / 2+\varepsilon^{\prime}} \leq \gamma_{2}\left(h_{\Gamma}\right)^{\varepsilon-\varepsilon^{\prime}}\|\phi\|_{-1 / 2+\varepsilon}(\Gamma) .
\end{gathered}
$$

Insertion into (4.4) then yields the result (4.2).

Remark. If one chooses $S^{h_{\Omega}}$ to be piecewise linear functions and $S^{h_{\Gamma}}$ to be piecewise constant then $k=k^{\prime}=2$. Taking $\varepsilon=1$ and $\varepsilon^{\prime}=0$ in (4.2) then yields $O(h)$ convergence for $w^{h}$ in $H_{1}(\Omega)$ and $\phi^{h}$ in $H_{-1 / 2}(\Gamma)$.

We turn now to the proof of (4.3), first for $\mathscr{A}$. As a first step we make a decomposition of $\mathscr{A}$. For the Green's function $g$ of (2.6) we write

$$
g(x, y)=\frac{i}{K} k_{0}(|x-y|)+m(x, y),
$$

where $K_{0}$ is the Bessel function of second kind with imaginary argument. This gives a corresponding decomposition of $\mathscr{S}$ and $S$ :

$$
\mathscr{S}[\phi]=\mathscr{S}_{0}[\phi]+\mathscr{S}_{1}[\phi], \quad S[\phi]=S_{0}[\phi]+S_{1}[\phi]
$$

in obvious notation. $K_{0}$ has the same singularity as $H_{0}^{(2)}$; hence the mapping properties of $S_{0}$ are the same as those for $S$. The kernel of $S_{1}$ is differentiable and hence $S_{1}$ maps $H_{s}(\Gamma)$ into $H_{s+2}(\Gamma)$. The crucial fact, an idea from [9], is the following.

LEMma 4.1. There exists a constant $\gamma>0$ such that $\left\langle\phi, S_{0}[\phi]\right\rangle \geq \gamma\|\phi\|_{-1 / 2}^{2}(\Gamma)$.

Proof: Consider $v=\mathscr{S}_{0}[\phi]$. This satisfies $\Delta v-v=0$ in $\Omega$ and $\Omega^{+}$and tends to zero exponentially as $|x| \rightarrow \infty$. We have $\phi=v_{n}^{+}-v_{n}^{-}$and $v^{+}=v^{-}=S_{0}[\phi]$. Applying Green's theorem to $\Omega$ and $\Omega^{\prime}$ (with a limiting argument) yields

$$
-\int_{\Omega}\left(|\nabla v|^{2}+v^{2}\right) d x+\int_{\Gamma} v_{n}^{-} v d s=0=-\int_{\Omega+}\left(|\nabla v|^{2}+v^{2}\right) d x-\int_{\Gamma} v_{n}^{+} v d s .
$$


Hence

$$
\int_{\Gamma} \phi S_{0}[\phi] d s=\int_{\Gamma}\left(v_{n}^{+}-v_{n}^{-}\right) v_{n} d s \leq-\int_{\Omega}\left(|\nabla v|^{2}+v^{2}\right) d x=-\|v\|_{1}^{2}(\Omega) .
$$

Now $v$ is a solution of $\Delta v-v=0$ in $\Omega$ with $v^{-}=S_{0}[\phi]$ on $\Gamma$. Hence $\phi=S_{0}^{-1}\left[v^{-}\right]\left(S_{0}^{-1}\right.$ is surjective as before). But regularity for the Dirichlet problem implies $\underline{\lambda}\left\|v^{-}\right\|_{1 / 2}(\Gamma) \leq$ $\|v\|_{1}(\Omega) \leq \bar{\lambda}\left\|v^{-}\right\|_{1 / 2}(\Gamma)$ for some $\underline{\lambda}, \bar{\lambda}$. Hence $\|v\|_{1}^{2}(\Omega) \geq \lambda\left\|v^{-}\right\|_{1 / 2}^{2}(\Gamma)=\underline{\lambda}\left\|S_{0}^{-1} \phi\right\|_{1 / 2}^{2}(\Gamma) \geq$ $\gamma^{\prime}\|\phi\|_{-1 / 2}^{2}$.

We now write, using (2.23),

$$
\mathscr{A}(U, V)=\mathscr{A}^{0}(U, V)+\mathscr{A}^{1}(U, V),
$$

where

$$
\begin{gathered}
A_{11}^{0}(w, v)=-\int_{\Omega}(\mu \nabla w \cdot \nabla \bar{v}+w \bar{v}) d x, \quad A_{11}^{1}(w, v)=\int_{\Omega}\left(\beta^{2}+1\right) w \bar{v} d x, \\
A_{12}^{0}(\phi, v)=A_{12}(\phi, v) ; \quad A_{21}^{0}(w, \psi)=\left\langle\bar{\psi}, \frac{1}{2} w^{-}\right\rangle, \quad A_{21}^{1}(w, \psi)=\left\langle\bar{\psi}, D\left[w^{-}\right]\right\rangle, \\
A_{22}^{0}(\phi, \psi)=-\left\langle\bar{\psi}, S_{0}[\phi]\right\rangle, \quad A_{22}^{1}(\phi, \psi)=-\left\langle\bar{\psi}, S_{1}[\phi]\right\rangle .
\end{gathered}
$$

We will now establish a weaker version of (4.3).

Lemma 4.2. There exists a constant $m^{\prime}>0$ such that for any $U=\{w, \phi\} \in \mathscr{H}_{0}$,

$$
\sup _{\substack{V \in \mathscr{H}_{0} \\ V \neq 0}}|\mathscr{A}(U, V)| \geq m^{\prime}\|U\|_{0}\|V\|_{0}
$$

Proof. Put $\tilde{U}=(w,-2 \phi)$. Then we seek a $V$ satisfying $(4.11)$ in the form $V=$ $\tilde{U}+\bar{M}, M=\{m, \psi\}$. We have

$$
\mathscr{A}(U, V)=\mathscr{A}^{0}(U, \tilde{U})+\mathscr{A}(U, \bar{M})+\mathscr{A}^{1}(U, \tilde{U}) .
$$

By (4.10) and Lemma (4.1),

$$
\begin{aligned}
\operatorname{Re} \mathscr{A}^{0}(U, \tilde{U}) & =\operatorname{Re} A_{11}^{0}(w, w)+\operatorname{Re}\left\{A_{12}(\phi, w)-2 A_{21}^{0}(w, \phi)\right\}-2 \operatorname{Re} A_{22}^{0}(\phi, \phi) \\
& =-\int_{\Omega}\left(\mu|\nabla w|^{2}+|w|^{2}\right) d x+2\left\langle\bar{\phi}, S_{0}[\phi]\right\rangle+\operatorname{Re}\{\langle\phi, \bar{w}\rangle-\langle\bar{\phi}, w\rangle\} \\
& \leq-k_{1}\|w\|_{1}(\Omega)^{2}-k_{2}\|\phi\|_{-1 / 2}^{2}(\Gamma)+\operatorname{Re}\{2 i \operatorname{Im}\langle\phi, \bar{w}\rangle\} \\
& \leq-k\|U\|_{0}^{2}
\end{aligned}
$$

We assert that we can choose $M$ so that

$$
\mathscr{A}(U, \bar{M})=-\mathscr{A}^{1}(U, \tilde{U}), \quad\|M\|_{0} \leq K\|U\|_{0} .
$$

If so, then we will have $\|V\|\left\|_{0} \leq\right\| U\|\|_{0}+K\|U\| \|_{0}$,

$$
|\mathscr{A}(U, V)| \geq k\left|\|U\|_{0}^{2} \geq \frac{k}{1+K}\right||U|\left\|_{0} \mid\right\| V \|_{0}
$$

which is (4.11).

We verify assertion (4.14). Define a functional $\mathscr{F}(Z)$ on $\mathscr{H}_{0}$ by

$$
\mathscr{F}(Z)=-\mathscr{A}^{1}(\bar{Z}, \tilde{U})
$$


and let $M$ be the solution of $\left(\mathrm{VP}_{1}\right)$ for this $\mathscr{F}$ :

$$
\mathscr{B}(M, Z)=\mathscr{F}(Z)=-\mathscr{A}^{1}(\bar{Z}, \tilde{U}) \text { for all } Z \in \mathscr{H}_{0} .
$$

We put $Z=\bar{U}$ in (4.16) and use (2.27) to obtain

$$
\mathscr{A}(U, \bar{M})=\mathscr{B}(M, \bar{U})=-\mathscr{A}^{1}(U, \bar{U})
$$

and we have the solution of (4.14).

Let.us study (4.16) more closely. Put $Z=\{z, \chi\}$. Then by (4.10)

$$
\begin{aligned}
\mathscr{F}(Z) & =-\mathscr{A}^{1}(\bar{Z}, \tilde{U})=-A_{11}^{1}(\bar{z}, w)-2 A_{21}^{1}(\bar{z}, \phi)-2 A_{22}^{1}(\bar{\chi}, \phi) \\
& =-\int_{\Omega}\left(\beta^{2}+1\right) \bar{z} \bar{w} d x-2\left\langle\bar{\phi}, D\left[\bar{z}^{-}\right]\right\rangle+2\left\langle\bar{\phi}, S_{1}[\bar{\chi}]\right\rangle \\
& =\langle F, \bar{z}\rangle+\left\langle p, \bar{z}^{-}\right\rangle+\langle\bar{\chi}, q\rangle,
\end{aligned}
$$

where

$$
F=-\left(\beta^{2}+1\right) w, \quad p=-2 N[\bar{\varphi}], \quad q=S_{1}[\bar{\varphi}] .
$$

If we compare (4.17) with (3.8) we see that the solution $M$ of $(4.16)$ is a solution of $\left(\tilde{\mathrm{P}}_{1}\right)$ with $(F, p, q)$ as in (4.18). Recall that our original assumption was $(w, \phi) \in \mathscr{H}_{0}$; hence $w \in H_{1}(\Omega)=H_{-1+2}(\Omega)$. Also, $\phi \in H_{-1 / 2}(\Gamma)$ so $p, q \in H_{3 / 2}(\Gamma)=H_{-1 / 2+2}(\Gamma)$. Thus Theoem 3.1 tells us that $M \in \mathscr{H}_{2}$ and

$$
\||M|\|_{2} \leq K\left(\|w\|_{1}(\Omega)+\|\phi\|_{-1 / 2}(\Gamma)\right)=K\|\mid U\|_{0} .
$$

The estimate (4.19) gives us the inequality in (4.14) but it also does more. It enables us to extend from Lemma 4.2 to the inequality (4.3) by approximation. Suppose we are given $\mathrm{U}^{h} \in \mathscr{H}_{0}$. We choose $V=\tilde{U}^{h}+M$ as above so that $\mathscr{A}\left(U^{h}, V\right) \geq m^{\prime}\left\|U^{h}\right\|_{0}^{2}$. The problem is that $M$ is not in $\mathscr{H}^{h}$ so we approximate it.

Let us assume that $k$ and $k^{\prime}$ in (A.1) and (A.2) are both greater than or equal to two. Then, since (4.19) implies $\|w\|_{2}(\Omega) \leq K\|U\|_{0}$ and $\|\psi\|_{1 / 2}(\Gamma) \leq K\|U\|_{0}$, we can find $m^{h_{\Omega}} \in S^{h_{\Omega}}$ and $\psi^{h_{\Gamma}} \in S^{h_{\Gamma}}$ such that

$$
\begin{gathered}
\left\|m-m^{h_{\Omega}}\right\|_{1}(\Omega) \leq \gamma_{1} K h_{\Omega}\|U\|_{0} ; \\
\left\|\psi-\psi^{h_{\Gamma}}\right\|_{-1 / 2}(\Gamma) \leq \gamma_{2} K h_{\Gamma}\|U\|_{0} .
\end{gathered}
$$

If we set $M^{h}=\left\{m^{h_{\Omega}}, \psi^{h}\right\}$ then we have

$$
\left\|M-M^{h}\right\|_{0} \leq c\left(h_{\Omega}+h_{\Gamma}\right)\|\| U \|_{0} .
$$

Now we have

$$
\begin{aligned}
\mid \mathscr{A}(U, \tilde{U}+ & \left.M^{h}\right)|=| \mathscr{A}(U, \tilde{U}+M)+\mathscr{A}\left(U, M^{h}-M\right) \mid \\
& \geq m^{\prime}|| U\left\|_{0}^{2}-m c \bar{A}\left(h_{\Omega}+h_{\Gamma}\right)|| U \mid\right\|_{0}^{2} \geq \frac{m^{\prime}}{2}\|U\| \|_{0}^{2} .
\end{aligned}
$$

if $h_{\Omega}$ and $h_{\Gamma}$ are sufficiently small. Moreover, we have

$$
\left\|\tilde{U}+M^{h}\right\|_{0} \leq\left|\|\tilde{U}\|_{0}+\|\| M\|\|_{0}+\|\| M-M^{h}\|\|_{0} \leq C^{\prime}\|\mid U\|_{0} ;\right.
$$

hence (4.22) yields (4.3)

The proof of (4.3) for the other problem is symmetric to the one just given and we omit it. 


\section{REFERENCES}

[1] Y. H. Pao and C. C. Mow, Diffraction of elastic waves and dynamic stress concentrations, Crane, Russak, New York, 1971

[2] F. J. Sánchez-Sesma, I. Herrera and J. Avilés, $A$ boundary method for elastic wave diffraction: application to scattering of SH waves by surface irregularities, Bull. Seism. Soc. Am. 72, 473-490 (1982)

[3] F. J. Sanchez-Sesma and J. A. Esquivel, Ground motion on alluvial valleys under incident plane SH waves, Bull. Seismolog. Soc. Amer. 29, 1107-1120 (1979)

[4] O. C. Zienkiewicz, D. W. Kelly and P. Bettes, The coupling of the finite element method and boundary solution procedures, Int. J. Numer. Methods in Eng. 11, 355-375 (1977)

[5] C. Johnson and J. C. Nedelec, On the coupling of boundary integral and finite element methods, Math. Computation 35, 1063-1079 (1980)

[6] R. C. MacCamy, Variational procedures for a class of exterior interface problems, J. Math. Anal. and Appl. 78, 248-266 (1980)

[7] A. K. Aziz and R. B. Kellogg, Finite element analysis of a scattering problem. To appear.

[8] G. Hellwig, Partial differential equations, Ginn-Blaisdell, Boston, 1964

[9] G. C. Hsiao and W. L. Wendland, A finite element method for some integral equations of the first kind, J. Math. Anal. and Appl. 58, 449-481 (1977)

[10] G. J. Fix, Hybrid finite element methods, in Numerical simulation of fluid motion (John Hoye, ed.) NorthHolland, Amsterdam, 1978

[11] J. L. Lions and E. Magenes, Non-homogeneous boundary value problems and applications, Springer-Verlag, New York, 1972 